EUROPEAN ORGANIZATION FOR NUCLEAR RESEARCH

European Laboratory for Particle Physics

CERN SL / 97-47 (BI)

LEP Energy Group

\title{
The Influence of Train Leakage Currents on the LEP Dipole Field
}

\author{
E. Bravin ${ }^{a}$, G. Brun ${ }^{a}$, B. Dehning ${ }^{a}$, A. Drees ${ }^{b}$, P. Galbraith ${ }^{a}$, \\ M. Geitz ${ }^{c}$, K. Henrichsen ${ }^{a}$, M. Koratzinos ${ }^{a}$, G. Mugnai $^{a}$
}

\begin{abstract}
The determination of the mass and the width of the $\mathrm{Z}$ boson at CERN's LEP accelerator, an $e^{+} e^{-}$storage ring with a circumference of approximately 27 kilometres, imposes heavy demands on the knowledge of the LEP counter-rotating electron and positron beam energies. The precision required is of the order of $1 \mathrm{MeV}$ or $\approx 20 \mathrm{ppm}$ frequency. Due to its size the LEP collider is influenced by various macroscopic and regional factors such as the position of the moon or seasonal changes of the rainfall in the area, as reported earlier. A new and not less surprising effect on the LEP energy was observed in 1995: railroad trains in the Geneva region perturb the dipole field. A parasitic flow of electricity, originating from the trains, travels along the LEP ground cable and the vacuum chamber, interacting with the dipole field. An account of the phenomenon with its explanation substantiated by dedicated measurements is presented.
\end{abstract}

to be submitted to Nuclear Instruments and Methods

August 22, 1997

\footnotetext{
a CERN, 1211 Geneva 23, Switzerland

$b$ BUGH Wuppertal, 42097 Wuppertal, Germany

${ }^{c}$ III. Physikalisches Institut, RWTH Aachen, Germany
} 


\section{Introduction}

The $\mathrm{Z}$ boson, which carries the weak neutral current, plays a central role in electroweak interactions $[1,2]$. The measurement of the mass and the width of the $\mathrm{Z}$ particle is one of the most important issues in the scientific program of LEP, CERN's Large Electron and Positron ring. Not least for series of high precision standard model tests, these two quantities ought to be known to the highest possible accuracy. For this purpose a $\mathrm{Z}$ resonance scan was performed in 1993 and again in 1995. The statistical accuracy, which can be achieved in the determination of the $\mathrm{Z}$ mass and width, is of the order of $1 \mathrm{MeV}$ when combining the statistics of the four LEP experiments. The systematic error arising from the uncertainty on the energy determination of LEP is common to all experiments and should be kept to similar orders of magnitude.

In general, measurements of the electron spin precession frequency provide the required knowledge of the beam energy with an intrinsic precision of better than $1 \mathrm{MeV}$ [3]. These very accurate measurements are periodically performed, typically a few times a week, and provide the absolute energy scale. To interpolate the beam energy evolution between two spin precession frequency measurements effects, which could cause energy changes, have to be controlled.

This article reports on a perturbation to the LEP dipole magnets which results in fast and slow drifts of the magnetic bending field of the order of a few $10^{-5}$ corresponding to a beam energy change of a few MeV. Within the task of the LEP beam energy determination such an effect cannot be neglected. The perturbations originate from a flow of electric current, which is caused by railroad trains in the Geneva region. The generation of the train leakage currents and the conduction mechanism in earth will be described. The field changes arising from train leakage currents have to be added to the already known variations due to temperature changes, terrestrial tides [4] and seasonal deformations of the LEP ring springing from the water level of the lake of Geneva and the amount of rainfall in the vicinity of CERN [5].

\section{The LEP Environment}

The LEP storage ring with approximately $27 \mathrm{~km}$ of circumference is situated at a depth of 65 to $120 \mathrm{~m}$ in the region between the Jura mountains and the Geneva area in proximity of two major railroad lines (figure 1). The $\mathrm{SNCF}^{1}$ route "Geneva - Lyon", via Bellegarde, operating at a voltage of $1500 \mathrm{~V}$ DC and the $\mathrm{CFF}^{2}$ route "Geneva - Lausanne" operating at $15000 \mathrm{~V}$ AC. The CFF runs another line to the Geneva airport. This line is isolated to ground to prevent any electric perturbation to the airport.

During both $\mathrm{Z}$ resonance scans, the LEP main bending field was monitored by two Nuclear Magnetic Resonance (NMR) probes installed in a reference dipole magnet on the surface, which is connected in series with the LEP tunnel dipole magnets. During the 1995 operation period the magnetic field was monitored at the first time directly in the LEP tunnel. Additional probes were installed in

\footnotetext{
${ }^{1}$ Societé Nationale des Chemins de Fer (French)

${ }^{2}$ Chemins de Fer Fédéraux (Swiss)
} 


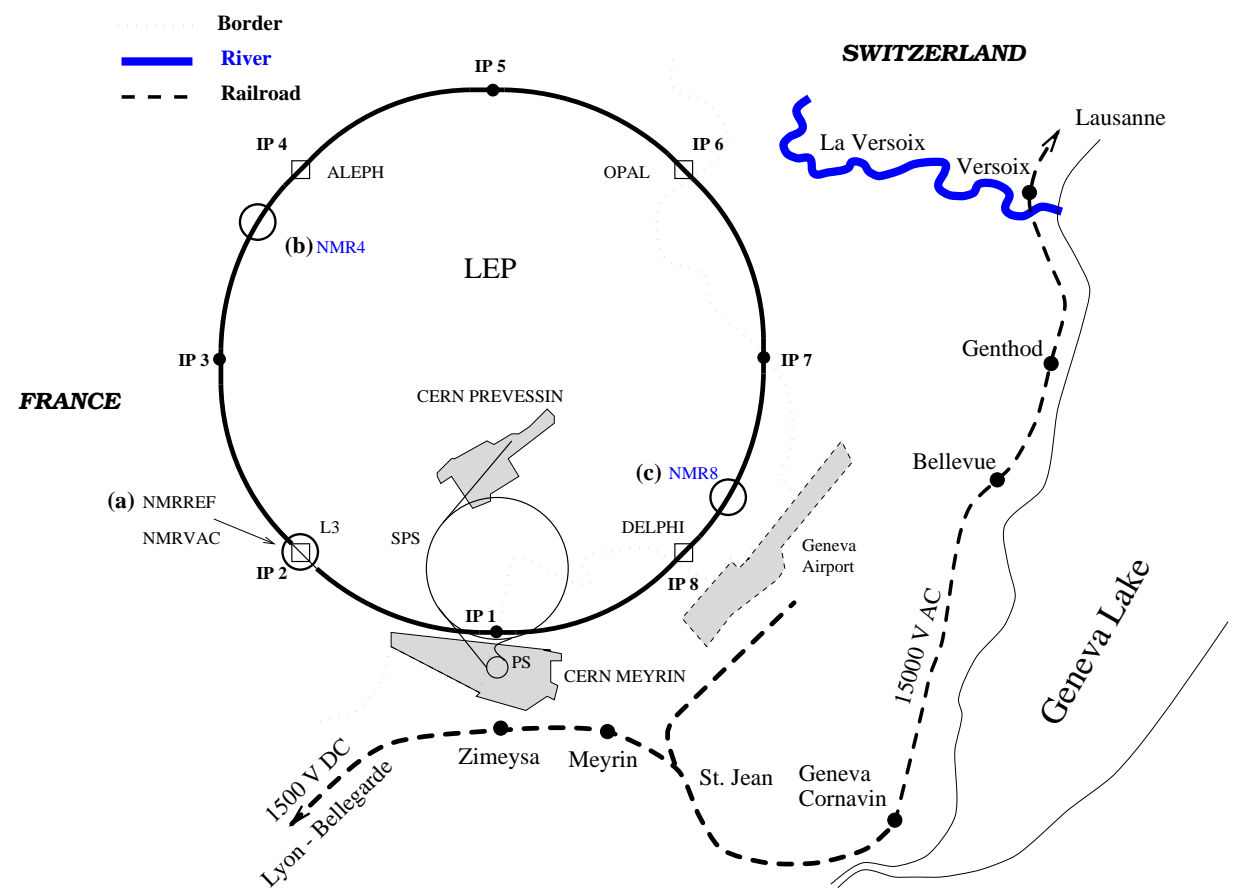

Figure 1: The LEP ring surrounded by the French and Swiss railroads with the locations of the NMR probes and the four experiments. Two probes (a) are installed in a reference dipole magnet, which is connected in series with the tunnel dipoles. NMR4 (b) and NMR8 (c) are mounted in tunnel magnets.

two of the tunnel dipoles. These two bending magnets are situated close to the Interaction Points IP4 and IP8 and the two probes are later referred to as the "tunnel probes" NMR4 and NMR8.

\section{LEP Dipole Field Evolution}

A LEP operation cycle (one 'fill') consists of a filling period during which electrons and positrons are accumulated at a low energy setting, an acceleration period to ramp to the nominal beam energy. Beam energy settings around the $\mathrm{Z}$ pole require a dipole field of about $500 \mathrm{G}$. The acceleration is followed by a 'physics' period with colliding beams during which the experiments record data. A complete LEP cycle, which is terminated by dumping of the beams, typically lasts for about 12 hours. Before LEP is refilled the dipole magnets are de-magnetised. Since spin precession frequency measurements require non-colliding beams, energy calibrations usually took place after the physics period.

During the 1995 LEP operation both short-term fluctuations (order of 1 second) and long-term rises (order of $1 \mathrm{~h}$ ) in the bending field up to $\Delta B / B \simeq 2-3 \cdot 10^{-5}$, corresponding to approximately $10-15 \mathrm{MeV}$, have been observed with the two tunnel probes $[6,7]$. In contrast to the tunnel probes the reference magnet probes would only register drifts of one order of magnitude smaller. Figure 2 shows a typical evolution of the bending field during an exemplary LEP fill. The increase of the magnetic field as a function of time fluctuates. It shows variations of the 


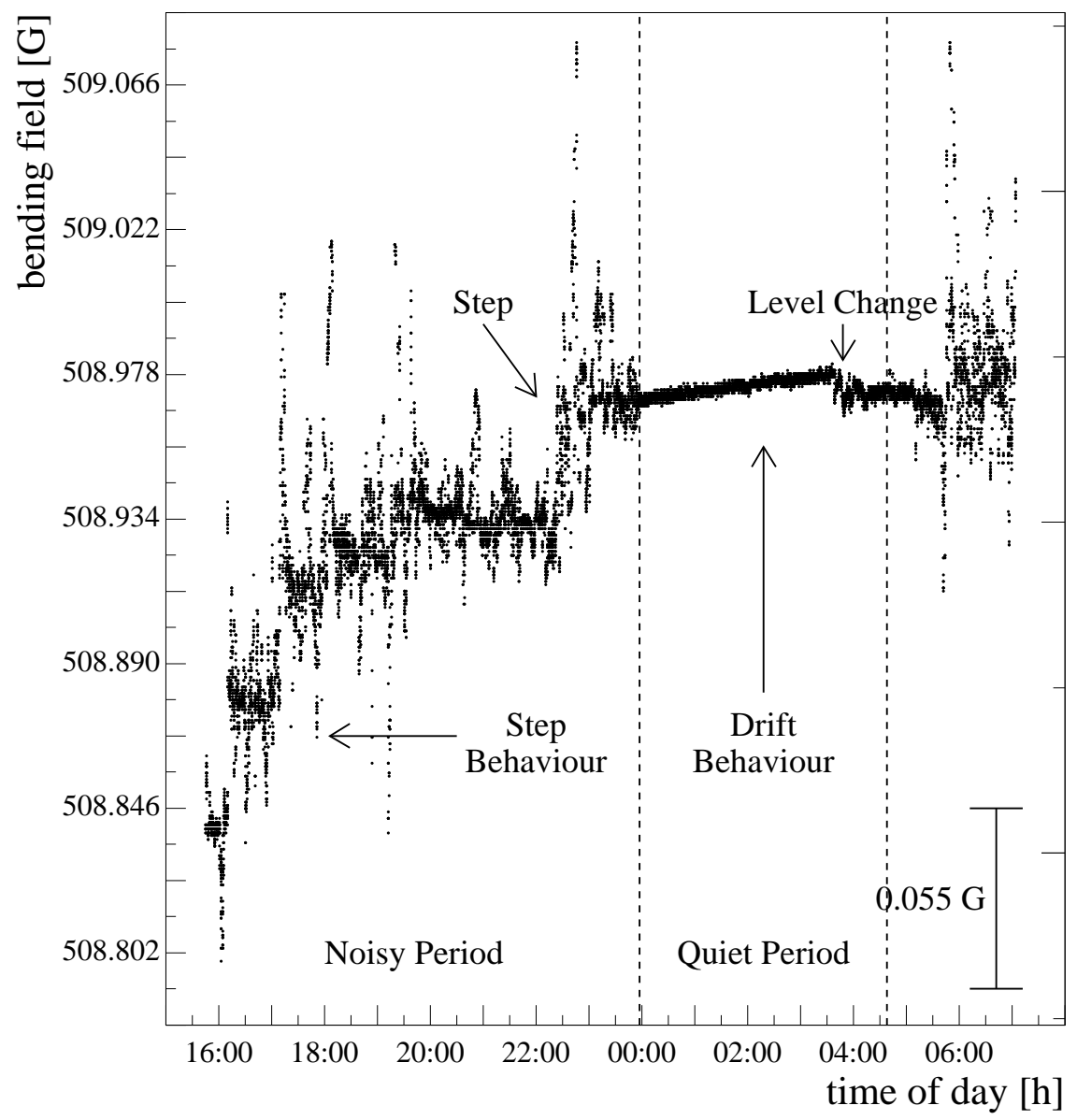

Figure 2: Magnetic field evolution measured in the tunnel by NMR8. The field increase during this period shows variations of the slope and steps of various sizes.

slope and steps of various sizes. The short term noise, mainly consisting of low frequency $(<1 \mathrm{~Hz})$ field fluctuations, shows a repetitive daily pattern of a quiet period between midnight and 04:30 and a noisy period outside this time interval. This pattern is present every day of a week. Figure 3 (bottom plot) shows more details of this typical noisy behaviour for a 15 minutes time interval during a later fill. The long-term rise seems preferentially be correlated with the noisy periods, where the short-term fluctuations act to increase the magnetic field.

\section{Train Leakage Currents}

\subsection{The Origin of the Field Changes}

The perturbation is attributed to leakage currents on the LEP vacuum chamber generated by trains circulating in the Geneva region. An example of a typical evolution of the train leakage current, the vacuum chamber current and the associated perturbation of the dipole magnetic field, measured in the tunnel by NMR8, is given in figure 3. The train leakage current is proportional to a potential difference measured between the railroad track and ground. The potential 


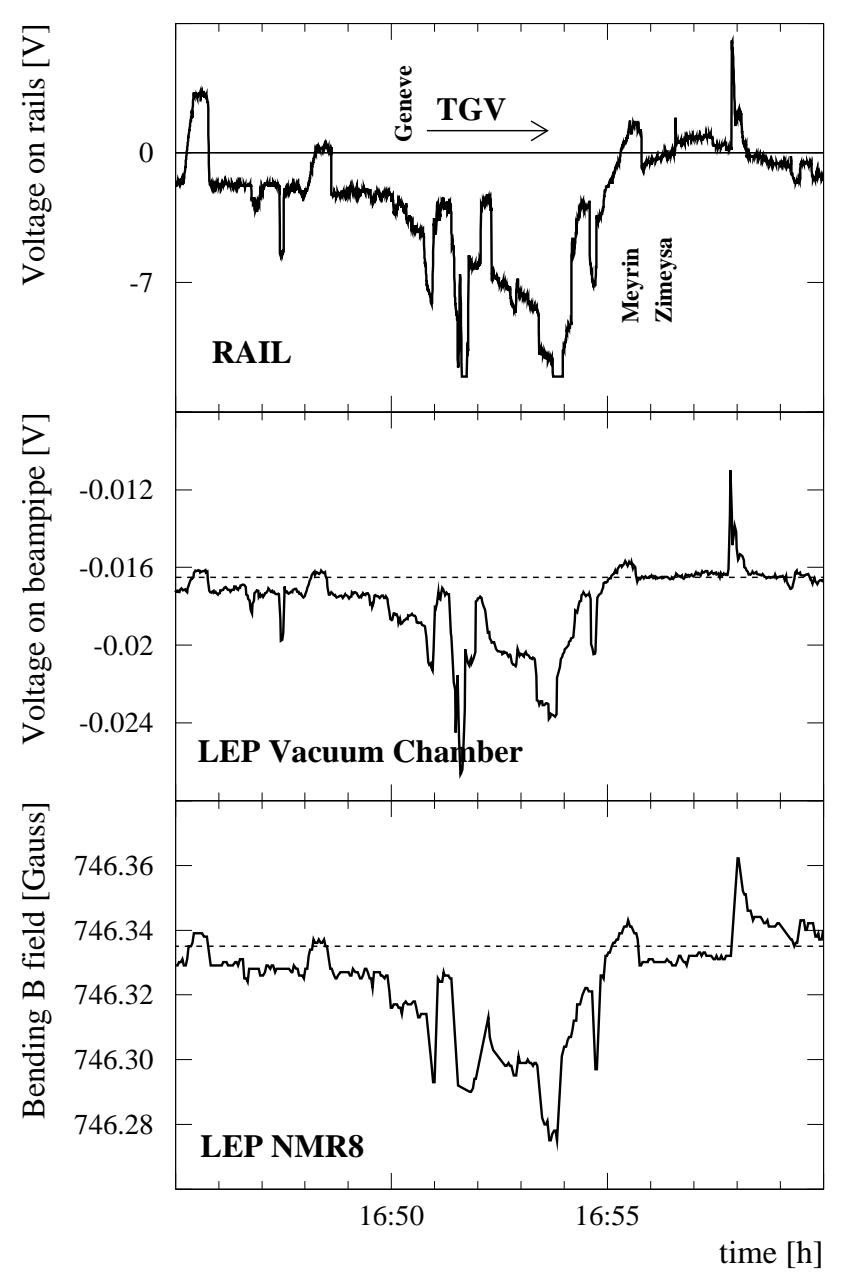

Figure 3: Train leakage currents, vacuum chamber currents and the associated magnetic field perturbation on Nov. 13th, 1995. The observed peaks are coincident with the departure of the 16:50 Geneva-Paris TGV (SNCF).

difference was measured at the Zimeysa train station on the Geneva-Lyon SNCF line. The vacuum chamber current is obtained by monitoring the voltage drop along a certain length of the beam pipe. The three distributions are obviously correlated. Additionally it could be observed that the current on the beam pipe is practically zero during the quiet period.

Therefore, it could be deduced that the parasitic current carried by the beam pipe affects the magnetic properties of the dipoles surrounding the vacuum chamber and creates the repetitive pattern of short-term fluctuations seen. However, the leakage current also induces long-term rises of the magnetic field $[6,7]$. The total amount of the induced drifts depends on the profile and the amplitude of the parasitic current as well as on the magnetic history of the dipoles: after a current spike the field of a magnet, which is not operated at the top of its hysteresis curve, does not drop to its initial value but to a value slightly higher. A second spike of the same magnitude at a later time would not further increase the field a larger spike would be needed instead. In average, the accumulated rise amounts 
to the order of a few MeV after a typical 10-hours fill.

In fact, field drifts are not exclusively caused by current peaks but also by the effect of temperature changes, which has the same order of magnitude [8]. Any rise seen during the quiet period, where only negligible leakage currents are flowing on the vacuum chamber, is due to temperature changes. Outside the quiet period and at the presence of temperature variations the overall rise is due to a convolution of either causes. Predictions under the assumption of a superposition of both effects agree reasonably well with the observed variations [8].

\subsection{The Propagation of DC Currents}

The excellent correlation between the field variations and the rail potential difference in figure 3 identifies the SNCF railroad line Geneva-Lyon to be the main culprit of the LEP dipole field perturbations. This French line is operated at $1500 \mathrm{~V}$ DC in contrast to the swiss CFF line, which is operated at $15000 \mathrm{~V}$ AC. The higher voltage of the CFF line reduces the electrical current consumption of CFF locomotives by a factor of 10 compared to an engine of a French SNCF train with the same power. Furthermore, the diffusion depth into earth of DC leakage currents is large while AC leaks are confined around their source ??. The Geneva-Airport railroad line, although operated at DC voltage too, is isolated to ground by construction.

Measurements of the DC parasitic currents along the LEP ground cable show how the leakage currents propagate. The leakage current was measured on the parallel ground cable which is electrically connected every $40 \mathrm{~m}$ to the vacuum chamber. Currents measured on the ground cable are therefore representative of the currents circulating along the vacuum chamber. The train leakage current captured by the LEP ground cable was measured simultaneously at the location of the NMR probe close to IP8 and at various places along the LEP tunnel. A measurement lasted about 160 minutes. Figure 4 shows the correlation factor as a function of the position along the LEP tunnel. A sign change of the correlation factor denotes a change of the direction of the current flow. Changes of the direction of the current flow on the ground cable identify the two points of the leakage current diffusion into LEP. These points are the electron injection line close to IP1 and a wide part of the octant between IP6 and IP7. At IP1 the CERN preaccelerators seem to catch the train leakage current and propagate it in the LEP tunnel. Leakage currents in the LEP tunnel circulate clockwise from IP1 via NMR4 towards IP6 and counterclockwise in the other branch. There, between IP6 and IP7, the Versoix river presumably provides a good local ground conductivity and the leakage current circuit is closed by the CFF line "Geneva - Lausanne" crossing the Versoix river as it can be seen in figure 1. The DC leakage current returns from the LEP tunnel to the rails and propagates along the railroads back to the rectifier stations.

\subsection{Generation and Conduction Mechanism}

Earth leakage currents are generated by railway engines [10]. An ideal railway supplies the electrical power via its top line while the circuit back to the rectifier station is closed by the rails. Due to rail isolation imperfections return currents 


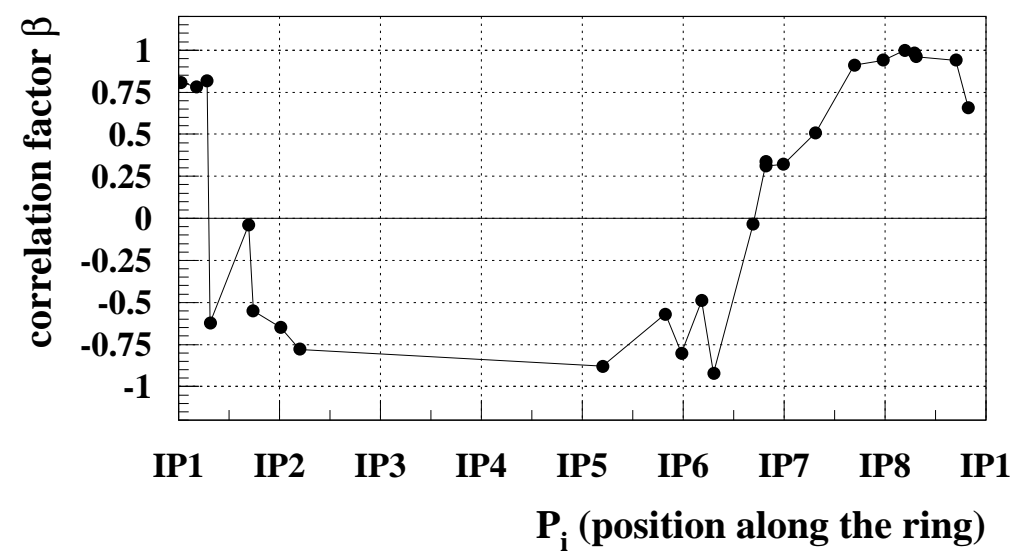

Figure 4: Correlation factor $\beta$ of the ground leakage currents in the LEP tunnel. The correlation between simultaneous ground current measurements at two positions along the ring is shown. One position is kept fixed at the NMR8 location and the second position $P_{i}$ is moved along the the vacuum chamber corresponding to the position marked on the horizontal axis. The sign of the correlation factor is inverted close to IP1 and IP6.

are able to leave this conductor and leak into the earth. Depending on the the resistivity, up to $25 \%$ [7] of the return current seeps into ground. Metal conductors, such as sewerage systems, pipes and wells are known to carry parasitic earth currents if the integral resistance of the path is minimised. The specific resistivity of the earth varies between 10 and $1000 \Omega \mathrm{m}$ and depends on temperature and moisture [9]. The conducting mechanism of currents within the earth can be described by electrolysis [11] or a parallel network of resistors. For the effect of the train leakage currents, the two poles involved are the rails on one hand and the rectifier station on the other hand (figure 5). The parallel network between the poles is formed by the preaccelerators (PS, SPS and injection line), the LEP tunnel, a region with local low specific resistivity, the railroad itself and earth. The resistance of earth with respect to the LEP tunnel is relatively high. Hence the path, which is most likely for the leakage current flow, follows the good conductors of the preaccelerators and the LEP tunnel. Topological environment, such as lakes or rivers - here the Versoix river -, are most decisive for local low specific resistivity [12]. However, leakage current variations arising from seasonal fluctuations of the specific resistivity, which would be expected from changes of the water flow in the Versoix river, could not be observed. 

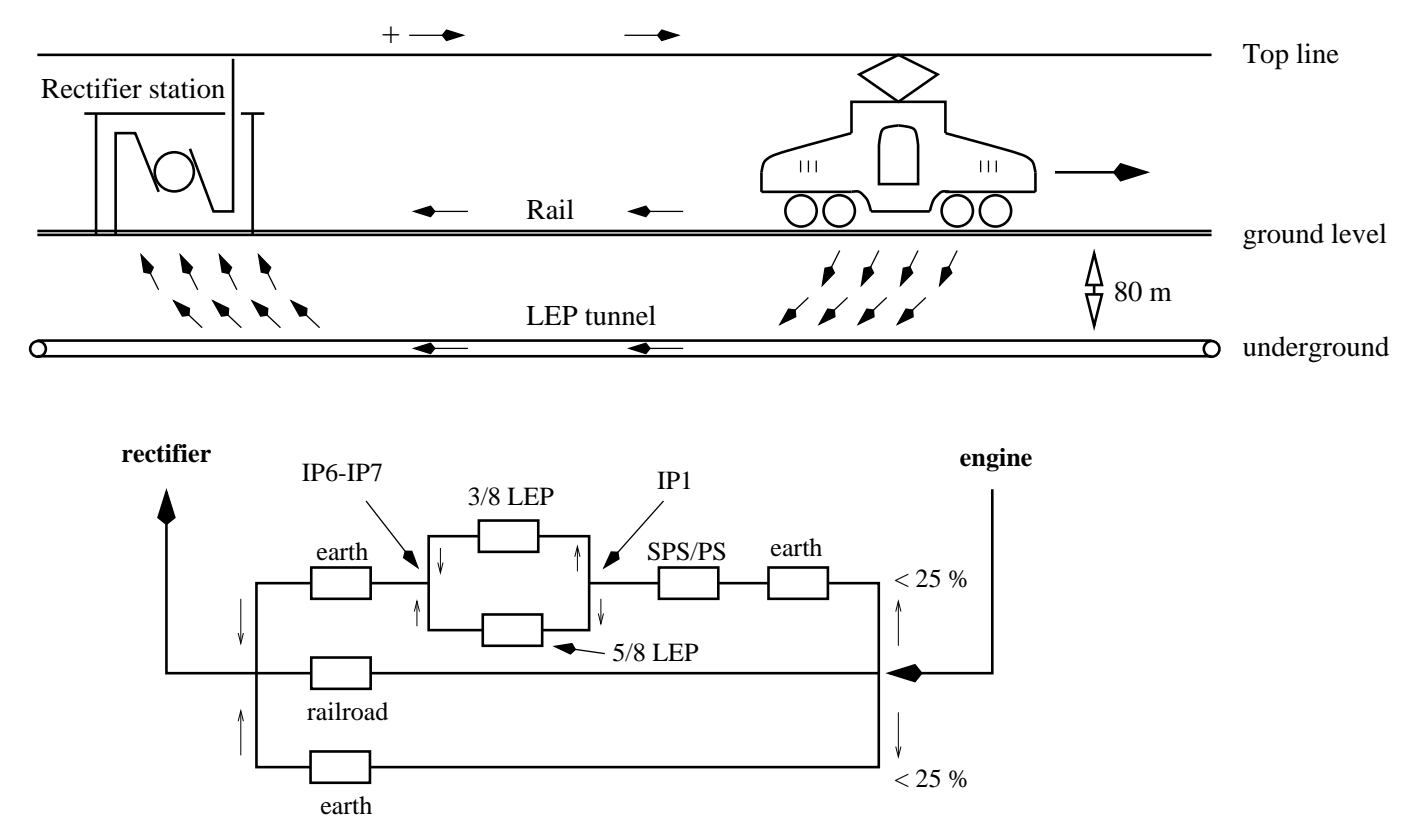

Figure 5: Schematic view of the train leakage current propagation. The upper part shows the actual path of the leakage currents at the presence of a good ground conductor (e.g. LEP). The lower part gives an equivalent representation of the electrical circuit. The small arrows indicate the flow of the leakage return current, which is less than $25 \%$.

The LEP tunnel is separated into two equivalent resistors representing two branches of $3 / 8$ and $5 / 8$ of the total circumference. The separation accounts for the difference in path length between IP1 and IP6 via NMR4 and NMR 8 respectively.

\section{Verification Measurement in the Laboratory}

The short-term and long-term effects of train leakage currents are considered to be reproducible for any representative magnet of the LEP bending dipole type, provided that the same current pattern is fed to the vacuum chamber inside the magnet. The reproducibility has been investigated on a dedicated test bench, which consisted of a LEP dipole, equipped with a short piece $(\simeq 1 \mathrm{~m})$ of the LEP vacuum chamber. The vacuum chamber was connected to a power supply capable of playing back a train leakage current profile stored on a disk. The original evolution of the voltage drop over a section of the beam pipe on Nov. 13th 1995 was saved and later fed back into the test bench power supply. The amplitude of the played-back current was adjusted to reproduce the same field changes as observed on Nov. 13th, 1995. Figure 6 shows the result of this simulation, performed on Jul. 9th, 1996. The correlation of the original magnetic field distribution (figure 6, top) and the magnetic field distribution from the simulation (figure 6, center) is remarkably good. The above correlation demonstrates that individual magnets respond in a very similar manner to vacuum chamber currents and allows some confidence in the understanding of the phenomenon. 


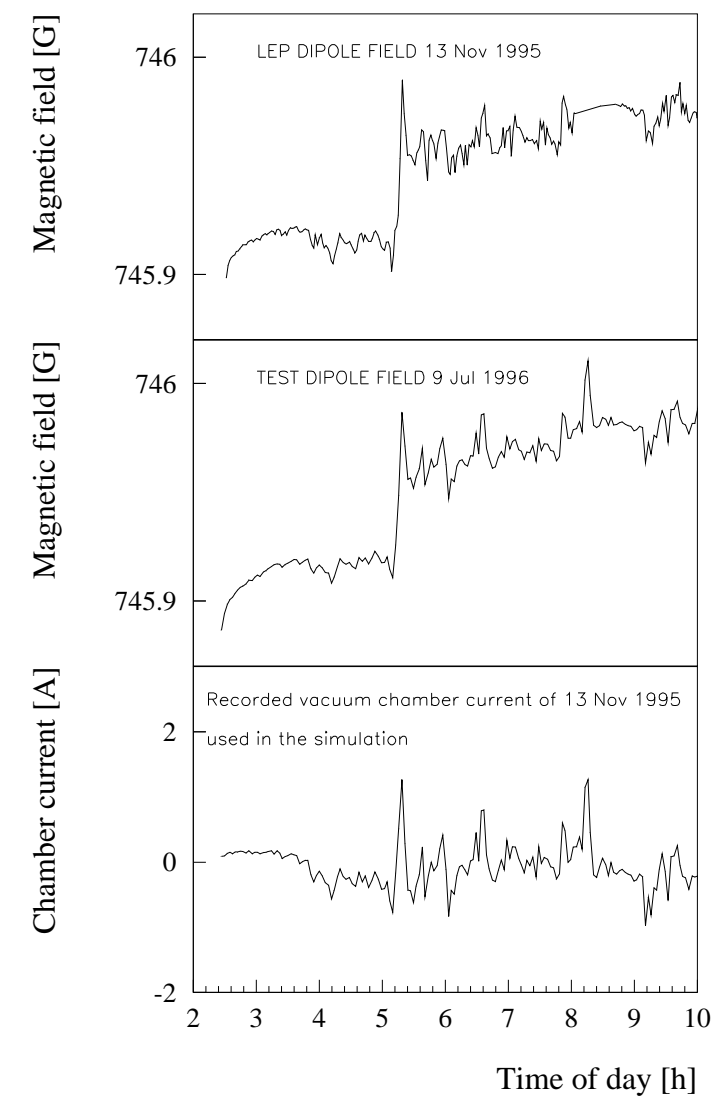

Figure 6: Magnetic field evolution measured with the NMR8 tunnel probe at Nov. 13th, 1995, (top) and the magnetic field in the test magnet during the simulation at Jul. 9th 1996 (center). The parasitic current evolution, which was recorded at Nov. 13th, 1995, is shown in the lower part of the figure. The start time of the simulation was normalised to the start time of the original data set. The large current spike around 05:15 causes a field jump in both, the original data and the simulation data.

\section{Conclusion}

Trains operating on SNCF rails in the Geneva area cause a flow of parasitic electric currents in the LEP tunnel and in particular along its vacuum chamber. The train leakage currents perturb the LEP bending field by producing both short-term fluctuations on a time scale of a few seconds as well as long-term field rises with a time constant of a few hours. The integrated field rise within a typical fill is of the order of $\Delta B / B \simeq 10^{-5}$, which corresponds to a beam energy change of some $\mathrm{MeV}$. The latter is a significant and non-negligible effect, which has to be taken into account for the systematic error calculation of the LEP energy to achieve the desirable accuracy of about $1 \mathrm{MeV}$.

\section{Acknowledgements}

We would like to thank everybody who helped in the challenge of explaining the phenomenon of the train leakage currents. This are in particular Mr. C. Fleury of $\mathrm{SIG}^{3}$ and Mr. J. M. Jolidon and assistants of the CCF. Mr. Fleury was the first one pointing out the trains as a possible source of the observed perturbation and provided equipment allowing the accurate measurement of the earth potential.

\footnotetext{
${ }^{3}$ Service Industriel Geneve
} 
He was the winner of a bottle of champagne, offered to anyone who had a hint unriddling the mysterious current flow on the LEP vacuum chamber. Mr. Jolidon kindly provided train schedules and made the rail potential measurements possible. We are indebted to the LEP operations team for the assistance and the patience in the difficult and never ending task of acquiring a better understanding of the LEP energy evolution and in particular of the magnetic dipole field behaviour.

\section{References}

[1] S. L. Glashow, Nucl. Phys. 22 (1961) 579;

A. Salam, Elementary Particle Theory, "Relativistic Groups and Analyticity", ed. N. Svartholm, Stockholm (1968);

S. Weinberg, Phys. Rev. Lett. 19 (1967) 1264.

[2] M. Veltman, Nucl. Phys. B 123 (1977) 89.

[3] L. Arnaudon et al., "Accurate Determination of the LEP Beam Energy by Resonant Depolarization", Z. Phys. C66 (1995) 45.

[4] L. Arnaudon et al.: "Effects of Terrestrial Tides on the LEP Beam Energy", Nucl. Instr. and Meth. A 357 (1995) 249-252.

[5] J. Wenninger, "Radial Deformations of the LEP ring", CERN SL/Note 95-21 (OP)

[6] B. Dehning, A. Drees and M. Geitz, "Study of the LEP Magnetic Dipole Field during the 1995 Energy Scan", CERN-SL/96-54 (BI) (1996).

[7] M. Geitz, "Investigation of Environmental Effects on the LEP Beam Energy - Magnetic Field Perturbations", Diplomawork, PITHA 96/14 (1996).

[8] A. Drees, "High Precision Measurements of the LEP Center-of-Mass Energies during the 1993 and 1995 Z Resonance Scans", PhD Thesis at the Bergische Universität und Gesamthochschule Wuppertal, WUB-DIS 97-5 (1997).

[9] R. Bräunlich, "Die messtechnische Überprüfung von grossen Erdungsanlagen", Bulletin SEV/VSE 23/95 (1995).

[10] F. J. Lowes, "Remote measurements of earth-leakage currents", IMechE 1990 C396/047 (1990).

[11] Societe technique et commerciale des canalisations souterraines en tubes d'acier (STECTA), "La protection cathodique des canalisations souterraines en acier", Paris (1957).

[12] H. Uhlig, "Corrosion et protection", Dunod, Paris, 1970. 